\title{
Dynamical theory: Application to spin-echo resolved grazing incidence scattering from periodic structures
}

\author{
Rana Ashkar, ${ }^{1,2, a)}$ W. L. Schaich, ${ }^{2}$ V. O. de Haan, ${ }^{3}$ A. A. van Well, ${ }^{3}$ R. Dalgliesh, ${ }^{4}$ \\ J. Plomp, ${ }^{3,4}$ and Roger Pynn ${ }^{1,2,5}$ \\ ${ }^{1}$ Indiana University Center for Exploration of Energy and Matter, Bloomington, Indiana 47408, USA \\ ${ }^{2}$ Physics Department at Indiana University, Bloomington, Indiana 47405, USA \\ ${ }^{3}$ Department of Radiation, Delft University of Technology, Mekelweg 15, $2629 \mathrm{JB}$ Delft, Netherlands \\ ${ }^{4}$ Rutherford Appleton Laboratory, Chilton, Oxfordshire OX11 OQX, United Kingdom \\ ${ }^{5}$ Neutron Science Directorate, Oak Ridge National Laboratory, Oak Ridge, Tennessee 37831, USA
}

(Received 1 October 2010; accepted 26 December 2010; published online 30 November 2011)

\begin{abstract}
Neutron spin-echo resolved grazing incidence scattering (SERGIS) measurements performed on a silicon diffraction grating with a rectangular profile were shown in our previous publications to be well explained by dynamical theory calculations. The theory is based on a Bloch wave expansion of the neutron wavefunction in the periodic layer of the grating, which includes all multiple scattering within that layer. Calculations show that the spin-echo polarization should be very sensitive to the scattering geometry (i.e., incident angle, sample alignment and beam divergence) and the sample specifications (i.e., grating period, groove depth). To test these predictions, SERGIS measurements have been performed on a set of gratings with different specifications in various scattering geometries. In all cases, simulations based on the dynamical theory, with all the parameters set to their known values, are in good agreement with the collected data. (C) 2011 American Institute of Physics. [doi:10.1063/1.3661162]
\end{abstract}

\section{INTRODUCTION}

Scattering techniques applied to surfaces and interfaces have evolved considerably in the past few decades due to the growing need to study samples with complicated profiles. Conventional probing techniques such as atomic force microscopy (AFM) or scanning electron microscopy are good for providing local surface and near surface information of the probed area. However, these contact probes cannot be used to characterize the morphology of structures buried deep under the free surface of the sample. Although a $3 \mathrm{D}$ reconstruction of the sample is possible through a layer by layer analysis accomplished, for example, by stepwise plasma etching and AFM measurements on the same area, ${ }^{1}$ this method results in the physical destruction of the sample. On the other hand, scattering techniques, combined with an accurate theoretical formulation are capable of retrieving profile information without any damage to the sample. In this regard, various theories have been proposed to extract information from such scattering measurements. Among these theories are the Born approximation, the distortedwave Born approximation, the phase-object approximation and the dynamical theory (DT). Although these theoretical approaches have given good account of data in several cases of electron, $\mathrm{x}$-ray and neutron scattering measurements, ${ }^{2-5}$ one should keep in mind that they do not hold for all scattering regimes. We are investigating the limits of validity of these theories and their applicability in various scattering regimes and will present our findings in a future work.

In this paper we discuss our ongoing study of two related aspects. On the experimental side, we explore the sensitivity

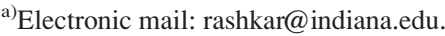

of a particular neutron scattering method (SERGIS ${ }^{6}$ ) to the details of both sample structure and scattering geometry. SERGIS measurements have been done on diffraction gratings with known profiles in different scattering configurations. Details of the experimental setup and results are discussed in Secs. III and IV, respectively. On the theoretical side, we demonstrate that a dynamical theory can accurately match the experimental data using only the known grating parameters. This is an important finding since sample morphology is often obtained by fitting a theoretical calculation to scattering data. If the theoretical formalism is approximate, then fitting can yield incorrect structural information. In Sec. II a brief discussion of our dynamical theory is given which complements the published derivation. ${ }^{7}$ Finally in Sec. V our conclusions are presented.

\section{DYNAMICAL THEORY}

The wavefunction, $\psi(\vec{r})$, of a neutron with an initial wavevector, $\overrightarrow{k_{0}}$, scattered from a sample with a scattering length density, $\rho(\vec{r})$, is a solution of the Schrödinger equation:

$$
\nabla^{2} \psi(\vec{r})+\left[k_{0}^{2}-4 \pi \rho(\vec{r})\right] \psi(\vec{r})=0 .
$$

The scattering geometry that we consider in this paper is one of grazing incidence where the neutron beam is incident on the grating almost parallel to its lines (which are along the $x$ axis as shown in Fig. 1). Due to translational invariance of the grating along the $x$ axis, the $x$ component of the wavevector transfer vanishes and we can effectively limit our study to scattering in the plane perpendicular to the free surface of the grating and its lines. Within this plane, we choose the $z$ axis ( $y$ axis) perpendicular (parallel) to the average surface of the grating (Fig. 1). To solve Eq. (1), the expression of the 


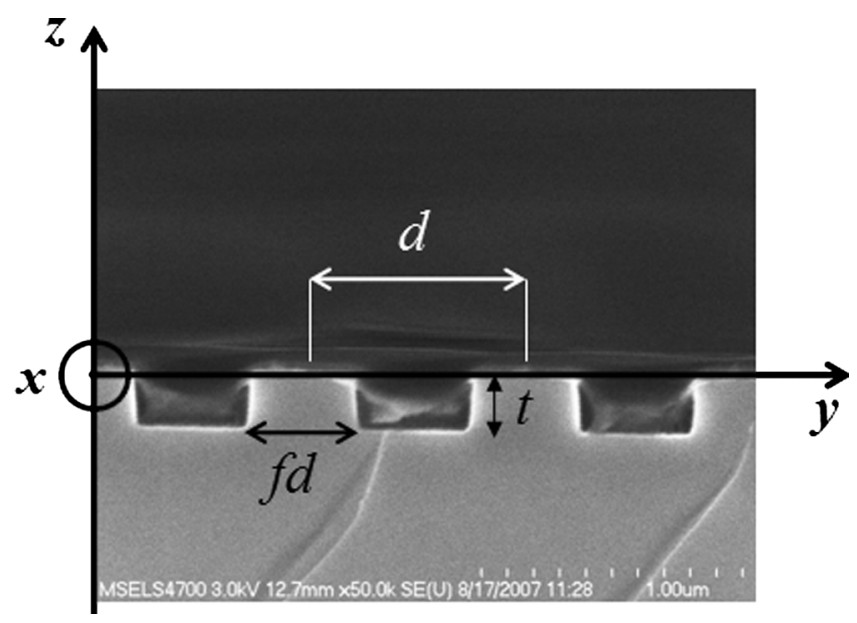

FIG. 1. SEM image of the grating indicating a rectangular profile with a period $d$, groove depth $t$, and filling factor $f$.

scattering length density must be known. With our choice of the coordinate axes we may write $\rho(\vec{r})$ as:

$$
\begin{array}{ll}
\rho(\vec{r})=\rho_{\text {air }}=0 & \text { for } \quad 0<z, \\
\rho(\vec{r})=\rho_{\text {mod }}(y)=\rho_{\text {mod }}(y+d) & \text { for } \quad-t<z<0, \\
\rho(\vec{r})=\rho_{\text {silicon }} & \text { for } \quad z<-t .
\end{array}
$$

Here $\rho_{\text {air }}$ and $\rho_{\text {silicon }}$ are the values of the neutron scattering length density in air and in the substrate respectively and $\rho_{\text {mod }}$ is the modulated scattering length density varying with

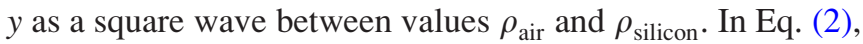
$t$ is the depth of the lines of the grating and $d$ is their period. The incident beam is a plane wave with wavevector components which, according to the scattering geometry shown in Fig. 2, can be expressed as:

$$
\begin{aligned}
& k_{0 x}=k_{0} \cos \theta \cos \varphi, \\
& k_{0 y}=k_{0} \cos \theta \sin \varphi, \\
& k_{0 z}=k_{0} \sin \theta .
\end{aligned}
$$

In our experiments, both $\theta$ and $\varphi$ are fractions of a degree.

First consider the form of the wavefunction in the air above the grating. In the region $z>0$

$$
\psi_{\text {air }}(\vec{r})=\left[e^{-i k_{0 z} z} e^{i k_{0 y} y}+\sum_{m} R_{m} e^{i k_{z} z} e^{i k_{y} y}\right] e^{i k_{0 x} x} .
$$

This is simply a sum of plane waves, since a plane wave is an eigenstate of the local free-space Hamiltonian. There is one plane wave propagating toward the surface (the incident beam with unit amplitude) and a set moving away from the surface, each with a separate reflection amplitude, $R_{m}$. The wavevector components of these reflected beams are $k_{x}=k_{0 x}, k_{y}=k_{0 y}+m g$ and $k_{z}=k_{z}(m)$. The first two relations follow from the wavevector conservation rules for our grating system: the $x$ component cannot be changed, while the $y$ component can only change by an integer multiple of the grating's smallest reciprocal lattice vector, $g=2 \pi / d$. The $z$ component is determined by energy conservation since the scattering process is elastic so all reflected beams have the same energy as the incident beam, i.e.,
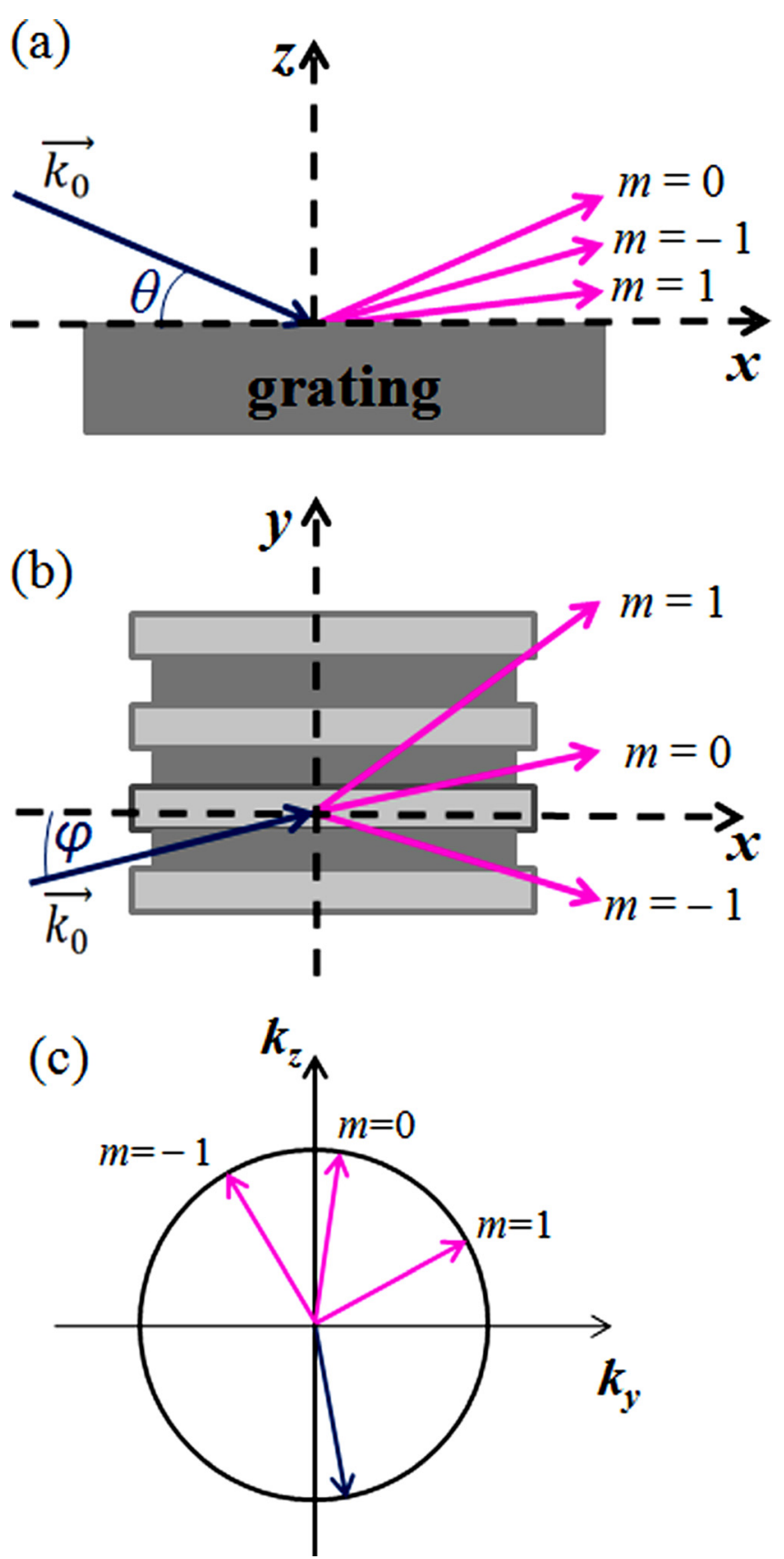

FIG. 2. (Color online) (a) and (b) Scattering geometry projected on the $x z$ plane and $x y$ plane, respectively. The neutron beam is incident on the grating with a grazing angle $\theta$ relative to the surface and an angle $\varphi$ relative to the lines. Beams are scattered with different angles depending on their diffraction orders, $m$. (c) Projection of the wavevectors above the grating onto the $k_{y}-k_{z}$ plane. The dark arrow represents the incident beam while the three light arrows (pink online) represent the reflected beams. This figure shows a specular beam $(m=0)$ and two (allowed) diffracted beams. The $y$ component of the wavevector difference between adjacent reflected beams is $q_{y}=g=2 \pi / d$. Since all the arrows are drawn from a common origin, their tips must lie on a circle for energy to be conserved.

$$
k_{z}(m)=\left[k_{0 z}^{2}+k_{0 y}^{2}-\left(k_{0 y}+m g\right)^{2}\right]^{1 / 2} .
$$

Note that $k_{z}^{2}(m)$ is positive only for a limited range of $m$. As long as it is positive, $k_{z}(m)$ is a (chosen positive) real number and the associated beam can propagate away from the surface. If $k_{z}^{2}(m)<0, k_{z}(m)$ is a (chosen positive) imaginary 
number and the beam decays away from the surface. For the latter case the beams are evanescent and, in the language of optics, contribute only to the "near field". They are important for the matching process between regions but do not reach the detector in the experiment.

For the propagating beams, the reflection coefficient is defined by: ${ }^{8}$

$$
\Re_{m}=\frac{k_{z}(m)}{k_{0 z}}\left|R_{m}\right|^{2}
$$

$\Re_{m}$ represents the fraction of the incident flux carried away by the $m$ th order reflected beam. An alternate characterization is that $\Re_{m}$ gives the relative probability for a neutron to be scattered with $y$ component of wavevector transfer $q_{y}=m g$.

This latter view allows contact with the theory of spinecho. These instruments are designed ${ }^{9}$ so that for a wavevector transfer $q_{y}$ the spin polarization of the scattered neutron is $\cos \left(q_{y} y_{\mathrm{se}}\right)$ where $y_{\mathrm{se}}$ is the spin-echo length defined in the next section. We can convert the relative probability $\Re_{m}$ to an absolute probability through the definition:

$$
\tilde{p}_{m}=\frac{\Re_{m}}{\sum_{m^{\prime}}^{\sim} \Re_{m^{\prime}}},
$$

where the $(\sim)$ sign indicates that only $m$ values of propagating reflected beams are included. Then it follows that the normalized spin-echo polarization, averaged overall possible reflections is given by

$$
\frac{P\left(y_{\mathrm{se}}\right)}{P_{0}}=\sum_{m} \tilde{p}_{m} \cos \left(m g y_{\mathrm{se}}\right),
$$

where $P$ and $P_{0}$ are the spin-echo polarizations obtained, respectively, by scattering from the sample and by reflecting from a smooth flat substrate. Hence one can easily calculate $P / P_{0}$ once the reflection amplitudes, $R_{m}$, are determined.

Our argument so far has not shown how $R_{m}$ can be found. This task is accomplished in dynamical theory by expansions of the neutron wavefunction in the other regions and a matching process. In the modulated layer, the neutron wavefunction is expressed as a sum of Bloch waves along the $y$ direction with corresponding plane wave factors along $+\hat{z}$ and $-\hat{z}$ (due to internal reflections at the interfaces). In the substrate, the neutron wavefunction is a sum of transmitted beams only. The reflection and transmission amplitudes of the neutron wavefunction in the different layers of the grating are determined by applying the condition that the wavefunction and its $z$ derivative are continuous at the interfaces of adjacent layers, i.e., at $z=0$ and $z=-t$. More details are given in our previous publication. ${ }^{7}$

We note here that to have a mathematically well-defined problem, the sums on $m$ in the three regions must run over the same values of $m$. We increase the range of the $m$ 's until the $R_{m}$ 's stop changing up to the third significant figure. For the present experiments this convergence of the dynamical theory (DT) usually sets in when $-5 \leq m \leq 5$; i.e., a total of 11 beams.

\section{SERGIS SETUP}

SERGIS measurements discussed in this paper were performed at the OFFSPEC beamline at ISIS. ${ }^{10}$ As in any spin echo apparatus, a polarizer at the beginning of the neutron flight path polarizes the neutron beam (say along $\hat{z}$ as shown in Fig. 3) which is then split by the first $\pi / 2$-flipper (to the left in Fig. 3) into two sub-beams with opposite spin states $|+z\rangle$ and $|-z\rangle$. The two sub-beams are guided through the different magnetic field regions (with the magnetic fields along the $z$ direction) into different paths dictated by the Zeeman interaction between the magnetic field and the spin state of the corresponding sub-beam. The $\pi$-flipper and the symmetry of the setup ensure that if the neutron beam is specularly reflected from the sample the relative phase acquired by the two sub-beams in the first half of the instrument is canceled in the second half. Consequently, when the two subbeams are recombined in the second $\pi / 2$-flipper (to the right of Fig. 3) the emerging neutron beam has the same polarization as the incident beam. This phenomenon of retrieving the initial polarization is called spin echo. If the scattering is not in the specular plane, the relative phase of the sub-beams is not totally canceled in the two parts of the instrument and a net phase is acquired by the emerging beam, resulting in a depolarization of the emerging beam. The normalized polarization measured in spin-echo experiments is determined by Eq. (8), where the spin echo length is given by:

$$
y_{\mathrm{se}}=2 \frac{\mu B}{E_{0}} L \cot (\alpha) .
$$

In Eq. (9), $L$ is the separation between the magnetic field regions with inclined boundaries, $\alpha$ is the angle between the inclined boundaries and the average neutron beam direction (Fig. 3), $E_{0}$ is the kinetic energy of the neutron beam in vacuum, and $\mu B$ is the Zeeman energy of a polarized neutron with a magnetic moment $\mu$ in the magnetic field, $B$, of the

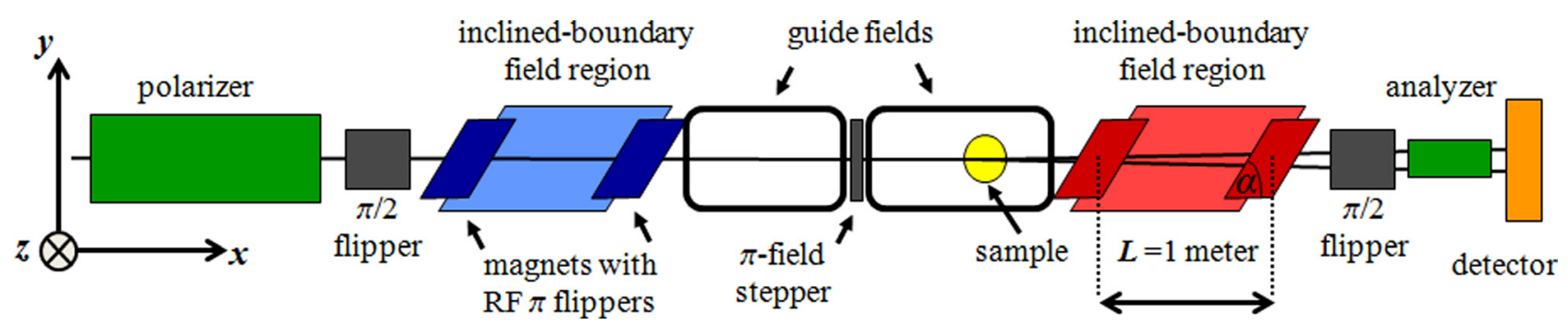

FIG. 3. (Color online) Schematic drawing of the OFFSPEC beamline at ISIS. The adjustability of the orientation of the inclined-boundary field regions allows for a wide range of the spin-echo length that can vary between few tens of nanometers and a few microns. 
inclined-boundary field regions. The spin echo length is effectively the distance over which correlations in the scattering sample are probed by the SERGIS technique.

In all the measurements described in this paper, except the one corresponding to Fig. 4, the angle of orientation of the inclined boundaries relative to incident neutron beam is set to $\alpha=79^{\circ}$. Substituting this value of the angle as well as the parameters of the instrument in Eq. (9) the relation of the spin-echo length to the neutron wavelength is found to be: $y_{\mathrm{se}}=\left(10^{3} \mathrm{~nm}^{-1}\right) \lambda^{2}$ with $y_{\mathrm{se}}$ and $\lambda$ both expressed in $\mathrm{nm}$. With this relation and with a neutron wavelength range $0.2 \mathrm{~nm}<\lambda<1 \mathrm{~nm}$, the spin echo length varies between $40 \mathrm{~nm}$ and $1 \mu \mathrm{m}$. For the data of Fig. 4, the relation is given by: $y_{\mathrm{se}}=\left(516 \mathrm{~nm}^{-1}\right) \lambda^{2}$, rendering a spin echo range of $\sim 20$ to $516 \mathrm{~nm}$.

\section{SENSITIVITY OF SERGIS}

Dynamical theory calculations on SERGIS geometries indicate that the spin-echo polarization is very sensitive to the scattering geometry and to the sample specifications. To test for this sensitivity we performed different SERGIS measurements on different samples varying one parameter at a time. In this paper, we will show the degree of sensitivity of SERGIS to the angle of grazing incidence, $\theta$; to the sample alignment defined by the sample alignment angle, $\varphi$, between the mean incident neutron beam and the lines of the grating; and to the height of the grating structures represented by the groove depth, $t$. It is important to note that the expression of the spin-echo polarization in Eq. (8) corresponds to a given value of $\theta$ and $\varphi$ within the incident beam divergence. Thus, to calculate the detected spin-echo polarization, $\left\langle P \mid P_{0}\right\rangle$, one should average Eq. (8) over the distribution of the incident $\theta$ and $\varphi$.

\section{A. Sensitivity to the angle of incidence}

In the first case, two grazing incidence measurements, with nominal average grazing angle $\theta_{0}=0.15^{\circ}$ or $\theta_{0}=0.18^{\circ}$,

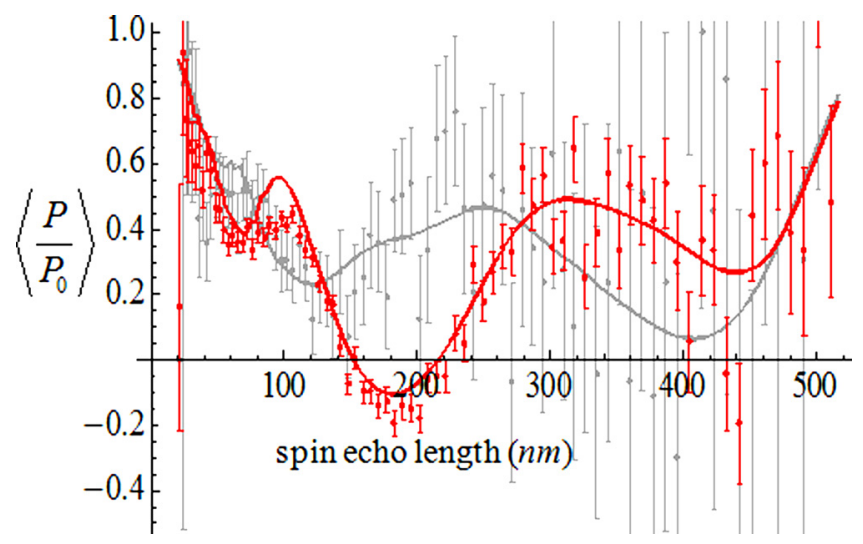

FIG. 4. (Color online) Plot of the spin echo polarization obtained from a grating with $d=556 \mathrm{~nm}, t=140 \mathrm{~nm}$, and $f=0.5$ perfectly aligned with the neutron beam $\left(\varphi_{0}=0^{\circ}\right)$ for (a) $\theta_{0}=0.15^{\circ}$ with the associated data (light, gray online) and (b) $\theta_{0}=0.18^{\circ}$ with associated data (dark, red online). The poor statistics in the former case is due to the short time of data collection. Solid lines are based on an 11-beam DT calculation with $y_{\mathrm{se}}=\left(516 \mathrm{~nm}^{-1}\right) \lambda^{2}$. were made on the same silicon grating of period $d=556 \mathrm{~nm}$, groove depth $t=140 \mathrm{~nm}$ and filling factor $f=0.5$ with the same (average) alignment angle $\varphi_{0}=0^{\circ}$. Even with such a small variation in the incident angle the spin-echo polarization differs dramatically as shown in Fig. 4. Dynamical theory simulations for these experiments have been done. For these (and all further) calculations the beam divergences in $\theta$ and $\varphi$ are represented by Gaussian distributions with a standard deviation of $0.01^{\circ}$ for $\theta$ and $0.1^{\circ}$ for $\varphi$, as calculated from slits widths and separations on the OFFSPEC instrument. The results show good agreement with the data in both cases of Fig. 4 and confirm the sensitivity of SERGIS to rather small differences in the angle of incidence, at least for the angles that we studied.

\section{B. Sensitivity to the sample alignment}

In the second case, a diffraction grating with $d=556 \mathrm{~nm}$, $t=110 \mathrm{~nm}$, and $f=0.5$ was measured with $\theta_{0}=0.15^{\circ}$, for two values of the alignment angle $\varphi: \varphi_{0}=0^{\circ}$ and $\varphi_{0}=0.3^{\circ}$.

For $\varphi_{0}=0^{\circ}$, the DT calculations are carried out with beams of orders between -5 and +5 . The choice of the truncation order is again determined by the smallest number of beams for which the polarization is found to converge. The choice of the symmetry in the included orders is due to the symmetric angular distribution of the incident beam around $\varphi_{0}=0^{\circ}$, which is a Gaussian centered at $\varphi_{0}=0^{\circ}$. In the calculation, we assume that all the reflected beams are captured by the detector. This assumption is justified by the fact that the scattered angles, $\varphi_{\text {scat }}$, lie within the angular acceptance of the detector as calculated from instrumental parameters: $|\delta \varphi|<0.4^{\circ}$, where $\delta \varphi$ is the deflection angle (away from $\left.\varphi_{0}\right)$ of the scattered beam. With these settings, the calculated spin echo polarization agrees with the measured polarization as shown in Fig. 5.

For the case where $\varphi_{0}=0.3^{\circ}$, the calculation is again based on an 11-beam approximation but the range is asymmetric with $-9 \leq m \leq 1$. This shift in the orders of the reflected beams included in the calculation is dictated by the

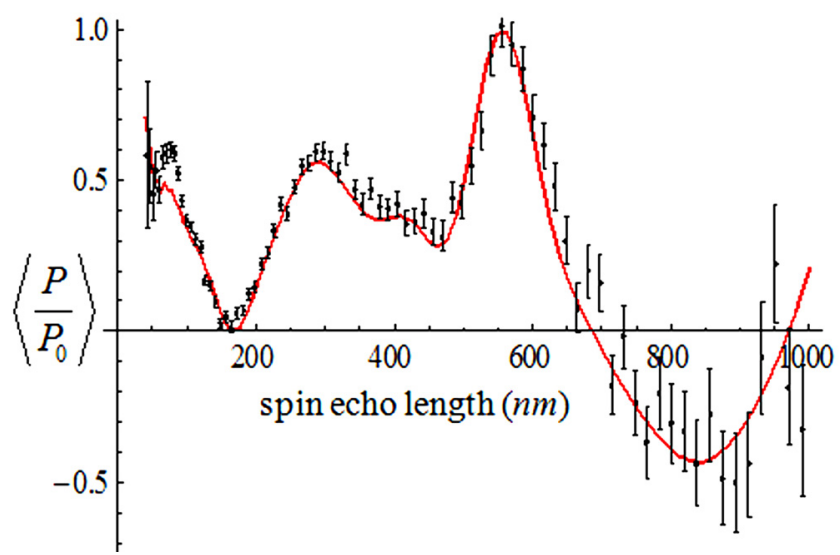

FIG. 5. (Color online) Plot of the spin echo polarization for a grating with $d=556 \mathrm{~nm}, t=110 \mathrm{~nm}$ and $f=0.5$ in the case of grazing incidence $\left(\theta_{0}=0.15^{\circ}\right)$ and perfect alignment with the neutron beam $\left(\varphi_{0}=0^{\circ}\right)$ obtained by an 11-beam DT calculation (dark line, red online) with all parameters set to experimentally observed values and $y_{\mathrm{se}}=\left(10^{3} \mathrm{~nm}^{-1}\right) \lambda^{2}$. Data is represented by black points with error bars. 
shift of the Gaussian distribution in $\varphi$, now centered at $\varphi_{0}=0.3^{\circ}$ instead of $\varphi_{0}=0^{\circ}$. Since the detector we used in our measurements has a limited angular acceptance in $\varphi$, not all the reflected beams can be captured by the detector and we need to restrict the values of $m$ appropriately. In Fig. 6, we show three calculations of the spin echo polarization for the same scattering geometry as the experiment: in the first we include all reflected beams; in the second we include only those beams scattered within the angular acceptance of the detector, considered to be centered with the incident beam (i.e., $-0.1^{\circ}<\varphi_{\text {scat }}<0.7^{\circ}$ ); in the third, we introduce a displacement of $+1 \mathrm{~cm}$ of the detector along the $y$ direction [cf. Figure 2(b)] so that $0.1^{\circ}<\varphi_{\text {scat }}<0.9^{\circ}$. The first calculation represented by a gray line in Fig. 6 obviously does not agree with the scattering data, whereas the second represented by the dashed line shows better agreement with the data. The third calculation shows the best match to the experimental results and is consistent with our knowledge of the azimuthal position of the detector, which we did not determine accurately enough during the experiment. This shift in the position of the detector causes beams of large wavelengths and large negative orders to be excluded in the calculations as they scatter outside the effective area of the detector. The remarkable differences between the three calculations illustrate the sensitivity of SERGIS to the number of beams that can be collected by the detector.

\section{Sensitivity to the groove depth}

For the case of sensitivity to the height of the studied structures, we intended to carry out two measurements with the same scattering geometry performed on two gratings that share all features except groove depth. The two gratings we used have the common values $d=556 \mathrm{~nm}$ and $f=0.5$, but one has $t=110 \mathrm{~nm}$ and the other $t=140 \mathrm{~nm}$. Unfortunately,

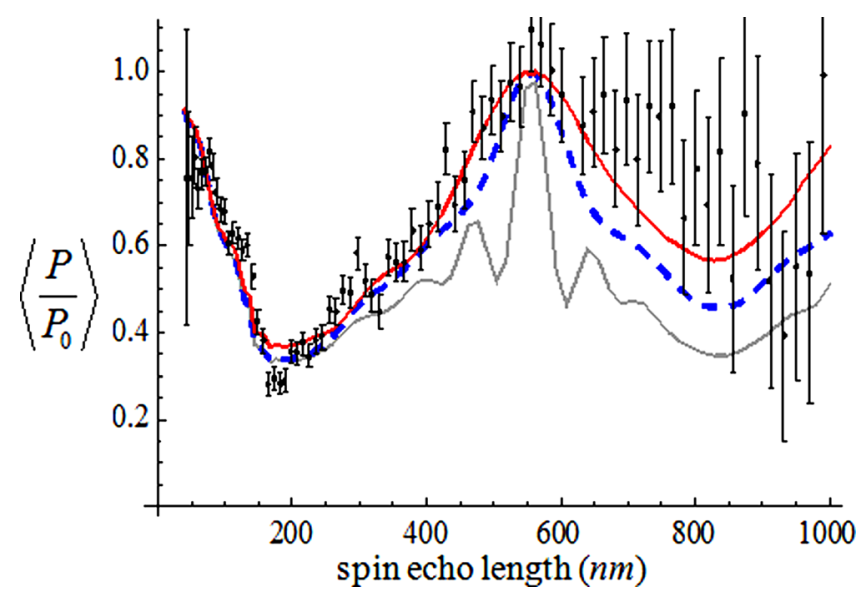

FIG. 6. (Color online) Plot of the spin echo polarization, with $y_{\mathrm{se}}=\left(10^{3} \mathrm{~nm}^{-1}\right) \lambda^{2}$, for the $t=110 \mathrm{~nm}$ grating in the case where the neutron beam is incident on the grating with an angle $\theta_{0}=0.15^{\circ}$ and at an angle $\varphi_{0}=0.3^{\circ}$. The gray line represents an asymmetric 11-beam DT calculation including all reflected beams. The other two lines are based on the same 11beam approximation but retain only beams within the angular acceptance of the detector. For the dashed line the detector is considered to be perfectly centered and for the solid line (red online) the center of the detector is displaced by $+1 \mathrm{~cm}$ (along the $y$ direction) relative to the incident beam.

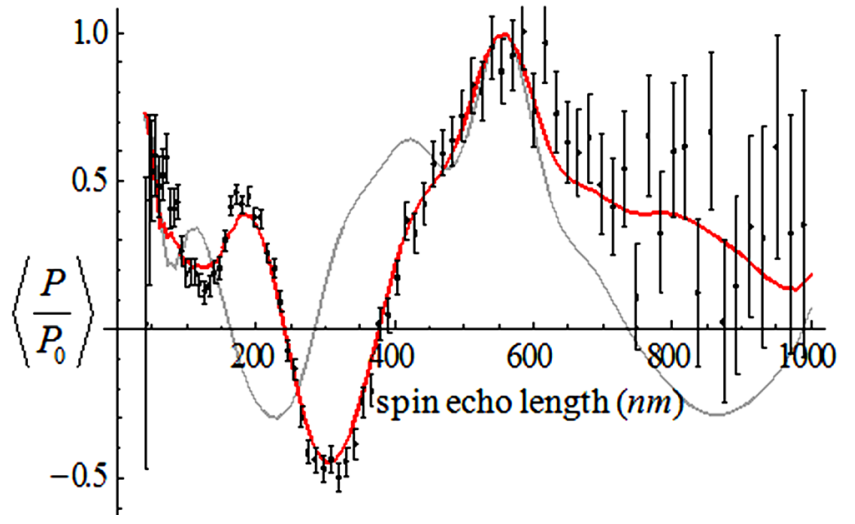

FIG. 7. (Color online) Dark line (red online): plot of the spin-echo polarization obtained by DT for the grating with $t=140 \mathrm{~nm}$ with $\theta_{0}=0.18^{\circ}$ and $\varphi_{0}=0^{\circ}$. The associated data is represented by black dots with error bars. Gray line: calculated spin-echo polarization for the grating with $t=110 \mathrm{~nm}$ with $\theta_{0}=0.18^{\circ}$ and $\varphi_{0}=0^{\circ}$. The two curves are based on an 11-beam approximation with the spin echo length given by: $y_{\mathrm{se}}=\left(10^{3} \mathrm{~nm}^{-1}\right) \lambda^{2}$.

$\theta_{0}$ was different in the two experiments. We later found that $\theta_{0}=0.18^{\circ}$ for the grating with $t=140 \mathrm{~nm}$ and $\theta_{0}=0.15^{\circ}$ for the grating with $t=110 \mathrm{~nm}$, so the two sets of data are not directly comparable because they differ in both groove depth and incident angle. However, DT calculations on the two gratings (with $t=110 \mathrm{~nm}$ or $t=140 \mathrm{~nm}$ ) for $\theta_{0}=0.18^{\circ}$, shown in Fig. 7, make it possible to predict the sensitivity of SERGIS to groove depth. We find that the normalized spin-echo polarization should easily pickup even a small change (30 $\mathrm{nm})$ in the height of the grating structures. Further, the data for the $t=140 \mathrm{~nm}$ grating presented in Fig. 7 shows the accuracy of the theory in reproducing the experimental results. It is important to note that although we did collect SERGIS data on the grating with the height $t=140 \mathrm{~nm}$ in a setting where $\theta_{0}=0.15^{\circ}$ as shown in Fig. 4, direct comparison with the data in Fig. 5 is not possible as these two sets of data have different $y_{\mathrm{se}} / \lambda^{2}$ ratios (discussed at the end of the previous section). This means that the same value of the spin-echo length in the two cases corresponds to two different values of the neutron wavelength. Knowing that the probabilities, $\tilde{p}_{m}$, are wavelength dependent, one cannot directly compare measurements done in different magnetic field settings.

\section{CONCLUSION}

The agreement between dynamical theory calculations and SERGIS measurements on gratings of known specifications shows the efficacy of this scattering technique in the determination of profile features with a considerable degree of accuracy. This is a first step in applying SERGIS, combined with an accurate theory, to extract profile information about in-plane periodic samples with unknown structures. The sensitivity of the SERGIS measurements to small variations in probed morphologies and the accuracy of DT in reproducing this sensitivity can be of great importance in several fields, such as the determination of self-assembled structures under confinement in the grooves of a grating. In fact, the sensitivity of the scattering technique and theoretical model to slight differences in the measured parameters plays a major role in the degree of accuracy with which these 
parameters can be extracted. In some cases, a rough estimate of the measured quantity can be enough for the scientific purposes behind the experiment, whereas in other cases a high degree of accuracy in determining specific features is required.

The dynamical theory simulation we have developed can be adapted for x-ray scattering from periodic structures. The theory can be also modified easily to apply to gratings with different profiles. In fact, we have developed a thinslicing dynamical theory code based on the Parratt formalism and it has been successfully implemented to reproduce neutron scattering data from gratings with trapezoidal and sinusoidal profiles.

\section{ACKNOWLEDGMENTS}

This work was supported by the U.S. Department of Energy through its Office of Basic Energy Sciences, Division of Material Science and Engineering (Grant No. DE-FG02-
09ER46279). We are grateful to the UK Science and Technology Facilities Council for the award of beam time.

${ }^{1}$ M. Konrad, A. Knoll, G. Krausch, and R. Magerle, Macromolecules 33, 5518 (2000).

${ }^{2}$ I. K. Robinson and D. J. Tweet, Rep. Prog. Phys. 55, 599 (1992).

${ }^{3}$ G. Renaud, R. Lazzari, and F. Leroy, Surf. Sci. Rep. 64, 255 (2009).

${ }^{4} \mathrm{~V}$. de Haan, Coherence Approach to Neutron Propagation in SpinEcho Instruments, 1st ed. (BonPhysics Research and Investigations Puttershoek, The Netherlands, 2007).

${ }^{5}$ C. J. Humphreys, Rep. Prog. Phys. 42, 1826 (1979).

${ }^{6}$ G. P. Felcher, S. G. E. teVelthuis, J. Major, H. Dosch, C. Anderson, K. Habicht, and T. Keller, Proc. SPIE 4785, 164 (2002).

${ }^{7}$ R. Ashkar, P. Stonaha, A. L. Washington, V. R. Shah, M. R. Fitzsimmons, B. Maranville, C. F. Majkrzak, W. T. Lee, W. L. Schaich, and R. Pynn, J. Appl. Crystallogr. 43, 455 (2010).

${ }^{8} \mathrm{~J}$. B. Pendry, Low Energy Electron Diffraction: The Theory and its Application to Determination of Surface Structure (Academic, New York, 1974).

${ }^{9}$ M. T. Rekveldt, J. Plomp, W. G. Bouwman, W. H. Kraan, S. Grigoriev, and M. Blaauw, Rev. Sci. Instrum. 76, 033901 (2005).

${ }^{10}$ J. Plomp, Doctoral Thesis, Delft University of Technology (2009). 